\title{
New Synthetic Routes to Acronycine, Noracronycine, and Their Analogues
}

\author{
Galla Sri Hari, Yong Rok Lee, ${ }^{\star}$ Xue Wang, ${ }^{\dagger}$ Won Seok Lyoo, ${ }^{\dagger}$ and Sung Hong Kim ${ }^{\S}$ \\ School of Chemical Engineering and Technology, Yeungnam University, Gyeongsan 712-749, Korea \\ ${ }^{*}$ E-mail: yrlee@yu.ac.kr \\ †School of Chemical Engineering \& Food Science, Xiangfan University, Hubei 441053, China \\ 'School of Textiles, Yeungnam University, Gyeongsan 712-749, Korea \\ ${ }^{\S}$ Analysis Research Division, Daegu Center, Korea Basic Science Institute, Daegu 702-701, Korea \\ Received June 18, 2010, Accepted June 28, 2010
}

Key Words: Acronycine, Noracronycine, 1,2-Dihydroacronycine, 1,2-Dihydronoracronycine

For a long time, acridone alkaloids have attracted much attention because of their broad spectrum of activity in several tumors, including sarcoma, myeloma, carcinoma, and melanoma. ${ }^{1}$ Acridone alkaloids have been also used as photosensitizers for cancer treatment. ${ }^{2}$ They are also have various biological properties, such as antiviral ${ }^{3}$ and antimalarial properties, ${ }^{4}$ induction of HL-60 cellular differentiation, ${ }^{5}$ and inhibition of Epstein-Barr virus activities. ${ }^{6}$ Among these alkaloids, acronycine (1) is isolated from the bark of Acronychia baueri ${ }^{7}$ and exhibits strong antitumor and antiproliferative activities on several tumor models, including leukemia, sarcoma, myeloma, carcinoma, and melanoma. ${ }^{8-9}$ Noracronycine (2) was isolated from Medicosma subsessilis (Figure 1). ${ }^{10}$ Various analogues have been synthesized and studied, and their structure-activity relationships have shown that the pyranyl ring is essential for the cytotoxic and antitumor properties. ${ }^{11}$ The attempts for improving the biological activity have been carried out by modifying the pyranyl ring through dihydroxylation ${ }^{12}$ and epoxidation. ${ }^{13}$ Its modified derivatives exhibited more potent antitumor activities than the original acronycine. ${ }^{14}$ Although several synthetic approaches have been described for acronycine and its derivatives, ${ }^{15}$ molecules with a long chain on the pyranyl ring have not been reported yet. A relationship between the activity and the presence of a long chain on the pyranyl ring has not been studied. Among known compounds, it was reported that the presence of the geranyl and prenyl groups leads to a remarkable increase in the corresponding bioactivities. ${ }^{16}$

\section{Results and Discussion}

Recently, biologically interesting natural products containing a pyranyl ring have been synthesized through a domino reaction in the presence of ethylenediamine diacetate (EDDA). ${ }^{17}$ In a continuous effort to synthesize biologically active molecules, a new and facile synthesis technique was investigated
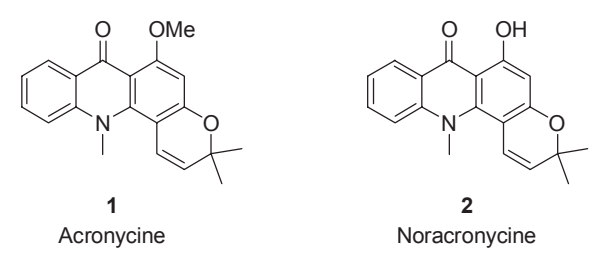

Figure 1. Naturally occurring acronycine (1) and noracronycine (2). for biologically interesting acronycine, noracronycine, and their derivatives. Thus, in this study a new synthetic route was reported for acronycine, noracronycine, and their derivatives with prenyl and geranyl groups.

First, a $65 \%$ yield of 1,3-dihydroxyacridone (4) was prepared as the starting material from phloroglucinol and anthranilic acid according to a known procedure (Scheme 1) in order to synthesize acronycine, noracronycine, and their derivatives. ${ }^{18}$

Acronycine, noracronycine, and their analogues were synthesized using the reaction in Scheme 2. Then $\mathbf{4}$ was reacted with 3-methyl-2-butenal in the presence of EDDA (20 mol \%) in THF under reflux for $8 \mathrm{~h}$ to produce the cyclized compound 5 at a yield of $85 \%$. Interestingly, in this reaction, the expected regioisomers were not detected. The assignment of 5 was confirmed through a comparison with the ${ }^{1} \mathrm{H}$ NMR data of the reported known compound. ${ }^{19}$ Acronycine (1) and noracronycine (2) were produced through the methylation of 5 . The treatment of $\mathbf{5}$ with an excess of methyl iodide in the presence of $\mathrm{K}_{2} \mathrm{CO}_{3}$ under reflux for $12 \mathrm{~h}$ produced 1 at a yield of $85 \%$, whereas the treatment with 1 equiv of methyl iodide at room temperature for $12 \mathrm{~h}$ provided 2 at a yield of $82 \%$. Then a hydrogenation reaction was carried out to produce 1,2-dihydroacronycine (6) and 1,2-dihydronoracronycine (7). The catalytic hydrogenation of 1 over $\mathrm{Pd} / \mathrm{C}(30 \mathrm{psi})$ in ethyl acetate for $1 \mathrm{~h}$ formed 6 at a yield of $80 \%$, whereas the hydrogenation of $\mathbf{2}$ for $1 \mathrm{~h}$ afforded 7 at a yield of $82 \%$.

Then $\mathbf{4}$ was further reacted with citral and trans,trans-farnesal in the presence of $20 \mathrm{~mol} \%$ of EDDA in order to synthesize the various analogues (Scheme 3). The reaction of $\mathbf{4}$ with citral and trans,trans-farnesal in refluxing THF produced $\mathbf{8}$ and $\mathbf{9}$ at yields of 80 and $70 \%$, respectively. Compounds 8 and 9 were methylated and hydrogenated in order to obtain their derivates. The reaction of $\mathbf{8}$ and $\mathbf{9}$ with methyl iodide in the presence of potassium carbonate in refluxing acetone for $2 \mathrm{~h}$ produced 10 and 11 at yields of 86 and $70 \%$, respectively. The catalytic

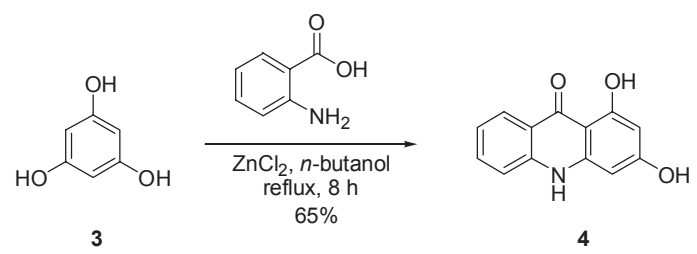

Scheme 1 

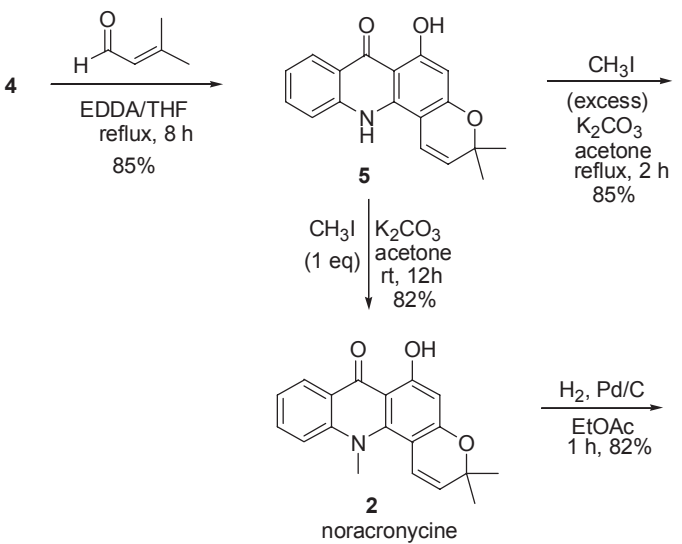
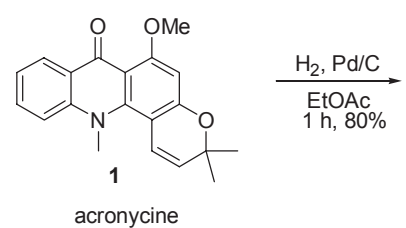

acronycine

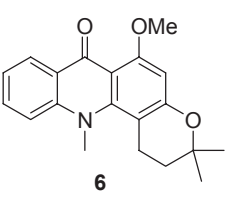

1,2-dihydroacronycine

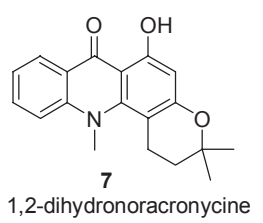

Scheme 2
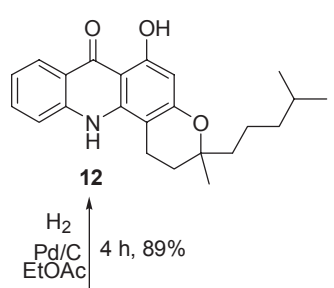
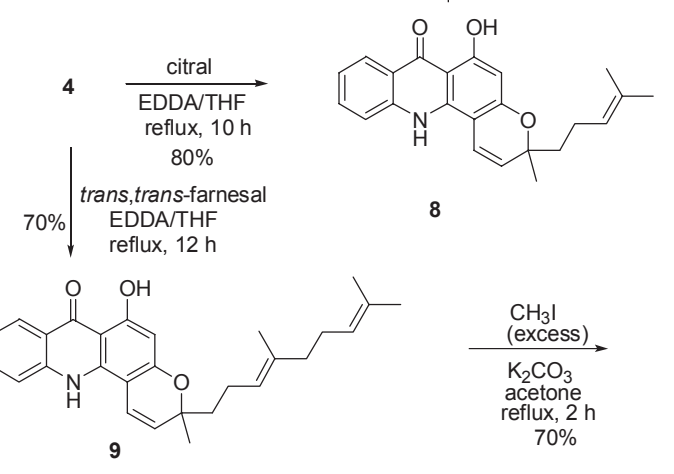

8
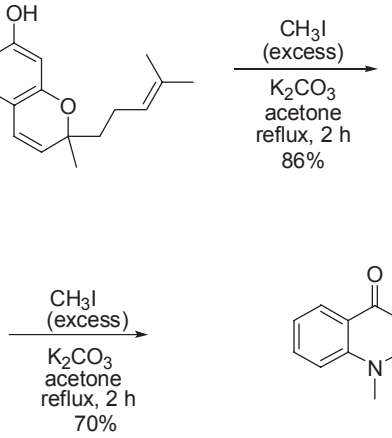

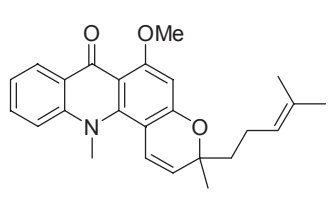

10

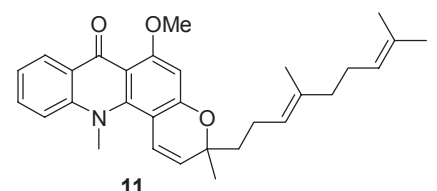

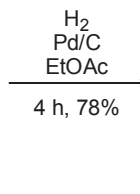

$\mathrm{Pd} / \mathrm{C}$
EtOAc
h, $78 \%$

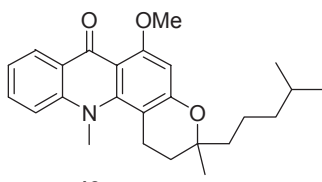

13 hydrogenation of 8 and $\mathbf{1 0}$ over $\mathrm{Pd} / \mathrm{C}$ (30 psi) provided $\mathbf{1 2}$ and 13 at yields of 89 and $78 \%$, respectively.

In conclusion, a concise and efficient synthetic route was described for biologically interesting acronycine, noracronycine, and their derivatives. These compounds were synthesized from acridone, which was prepared from commercially available phloroglucinol and anthranillic acid. Further work on the influence of the acronycine and noracronycine derivatives with a long chain is currently in progress for biological activities.

\section{Experimental}

All of the reactions were conducted under a nitrogen atmosphere. Merck precoated silica gel plates (Art. 5554) with a fluorescent indicator were used for the TLC analysis. Flash column chromatography was performed using silica gel 9385 (Merck, City, State, Country). The ${ }^{1} \mathrm{H}$ and ${ }^{13} \mathrm{C}$ NMR spectra were recorded using a Bruker Model ARX ( 300 and $75 \mathrm{MHz}$, respectively) spectrometer in $\mathrm{CDCl}_{3}$, DMSO- $d_{6}$ and acetone- $d_{6}$ as the solvent for the chemical shift. The IR spectra were recorded using a Jasco FTIR 5300 spectrophotometer. The HRMS and MS spectra were carried out at the Korea Basic Science
Institute.

Compound 4. A mixture of anthranilic acid $(6.85 \mathrm{~g}, 50 \mathrm{mmol})$, phloroglucinol (3) (6.85 g, $50 \mathrm{mmol})$, anhydrous $\mathrm{ZnCl}_{2}(7 \mathrm{~g}$, $51 \mathrm{mmol})$ and $n$-butanol $(150 \mathrm{~mL})$ was refluxed for $8 \mathrm{~h}$. The mixture was cooled, diluted with benzene-water $(1: 1,100 \mathrm{~mL})$, the organic layer was separated and dried over anhydrous $\mathrm{Na}_{2}$ $\mathrm{SO}_{4}$. Removal of solvents under reduced pressure left an oily mass which was dissolved in hot and aqueous $\mathrm{NaOH}(5 \%, 500$ $\mathrm{mL})$ and filtered. The filtrate on acidification with dil. $\mathrm{H}_{2} \mathrm{SO}_{4}$ (5\%) afforded product, which was purified by column chromatography on silica gel with hexane/EtOAc (2:1) to give 4 ( $7.4 \mathrm{~g}$, $65 \%$ ) as greenish yellow solid. mp $318-320{ }^{\circ} \mathrm{C}$. ${ }^{1} \mathrm{H}$ NMR (300 MHz, Acetone- $\left.d_{6}\right) \delta 10.70(1 \mathrm{H}, \mathrm{s}), 8.25(1 \mathrm{H}, \mathrm{d}, J=8.1 \mathrm{~Hz})$, 7.71-7.65 $(1 \mathrm{H}, \mathrm{m}) 7.46(1 \mathrm{H}, \mathrm{d}, J=8.4 \mathrm{~Hz}), 7.28-7.22(1 \mathrm{H}, \mathrm{m})$, $6.33(1 \mathrm{H}, \mathrm{d}, J=2.1 \mathrm{~Hz}), 6.10(1 \mathrm{H}, \mathrm{d}, J=2.1 \mathrm{~Hz})$.

Compound 5. To a solution of $4(567.5 \mathrm{mg}, 2.5 \mathrm{mmol})$ in THF $(10 \mathrm{~mL})$ was added 3-methyl-2-butenal $(630 \mathrm{mg}, 7.5 \mathrm{mmol})$, EDDA ( $90 \mathrm{mg}, 0.5 \mathrm{mmol}$ ) and the reaction mixture was refluxed under nitrogen atmosphere for $8 \mathrm{~h}$. Removal of the solvent at reduced pressure left dark colored semi solid, which was then purified by column chromatography on silica gel with hexane/ EtOAc (10:1) to give $5(623 \mathrm{mg}, 85 \%)$ as yellow solid. mp 248 - 
$249{ }^{\circ} \mathrm{C} .{ }^{1} \mathrm{H}$ NMR $\left(300 \mathrm{MHz}, \mathrm{CDCl}_{3}+\mathrm{DMSO}-d_{6}\right) \delta 8.21(1 \mathrm{H}$, $\mathrm{d}, J=7.8 \mathrm{~Hz}), 7.70(1 \mathrm{H}, \mathrm{d}, J=8.4 \mathrm{~Hz}), 7.59(1 \mathrm{H}, \mathrm{t}, J=7.5 \mathrm{~Hz})$, $7.19(1 \mathrm{H}, \mathrm{t}, J=7.5 \mathrm{~Hz}), 7.01(1 \mathrm{H}, \mathrm{d}, J=9.9 \mathrm{~Hz}), 6.05(1 \mathrm{H}, \mathrm{s})$, $5.54(1 \mathrm{H}, \mathrm{d}, J=10.2 \mathrm{~Hz}), 1.44(6 \mathrm{H}, \mathrm{s}) .{ }^{13} \mathrm{C} \mathrm{NMR}(75 \mathrm{MHz}$, $\left.\mathrm{CDCl}_{3}+\mathrm{DMSO}-d_{6}\right) \delta 180.8,163.9,159.3,140.7,137.7,133.1$, 125.0, 124.7, 121.2, 119.1, 117.0, 116.0, 104.2, 97.8, 96.4, 76.6, 27.4. IR (KBr) 3331, 2942, 1645, 1540, 1475, 1428, 1331, 1154, $757 \mathrm{~cm}^{-1}$. HRMS $\mathrm{m} / z(\mathrm{M})^{+}$calcd for $\mathrm{C}_{18} \mathrm{H}_{15} \mathrm{NO}_{3}: 293.1052$. Found: 293.1050.

Acronycine (1). To a solution of 5 (293 mg, $1 \mathrm{mmol})$ in acetone $(15 \mathrm{~mL})$ was added iodomethane $(1.42 \mathrm{~g}, 10 \mathrm{mmol})$, potassium carbonate $(1.38 \mathrm{~g}, 10 \mathrm{mmol})$ and the reaction mixture was refluxed under nitrogen atmosphere for $2 \mathrm{~h}$. The reaction mixture was filtered and removal of the solvent at reduced pressure left yellow colored solid, which was then purified by column chromatography on silica gel with hexane/EtOAc (1:1) to give 1 (273 mg, 85\%) as yellow solid. mp $174-175{ }^{\circ} \mathrm{C} .{ }^{1} \mathrm{H}$ NMR $\left(300 \mathrm{MHz}\right.$, Acetone- $\left.d_{6}\right) \delta 8.16(1 \mathrm{H}, \mathrm{d}, J=8.1 \mathrm{~Hz}), 7.62$ $(1 \mathrm{H}, \mathrm{m}), 7.48(1 \mathrm{H}, \mathrm{d}, J=8.7 \mathrm{~Hz}), 7.19(1 \mathrm{H}, \mathrm{m}), 6.69(1 \mathrm{H}, \mathrm{d}$, $J=9.6 \mathrm{~Hz}), 6.29(1 \mathrm{H}, \mathrm{s}), 5.57(1 \mathrm{H}, \mathrm{d}, J=9.6 \mathrm{~Hz}), 3.86(3 \mathrm{H}, \mathrm{s})$, $3.84(3 \mathrm{H}, \mathrm{s}), 1.49(6 \mathrm{H}, \mathrm{s}) .{ }^{13} \mathrm{C}$ NMR $\left(75 \mathrm{MHz}\right.$, Acetone- $\left.d_{6}\right) \delta$ 176.4, 163.7, 160.0, 147.7, 145.7, 133.2, 127.1, 126.4, 123.8, $122.8,117.6,111.2,104.1,95.0,76.9,56.3,44.8,27.0$. IR $(\mathrm{KBr})$ 2970, 1634, 1597, 1496, 1395, 1326, 1206, 1131, 1037, 778 $\mathrm{cm}^{-1}$. HRMS $m / z(\mathrm{M})^{+}$calcd for $\mathrm{C}_{20} \mathrm{H}_{19} \mathrm{NO}_{3}: 321.1365$. Found: 321.1363 .

Noracronycine (2). To a solution of $\mathbf{5}(293 \mathrm{mg}, 1 \mathrm{mmol})$ in acetone $(15 \mathrm{~mL})$ was added iodomethane $(142 \mathrm{mg}, 1 \mathrm{mmol})$, potassium carbonate $(138 \mathrm{mg}, 1 \mathrm{mmol})$ and the reaction mixture was stirred at $\mathrm{rt}$ under nitrogen atmosphere for $12 \mathrm{~h}$. The reaction mixture was filtered and removal of the solvent at reduced pressure left yellow colored solid, which was then purified by column chromatography on silica gel with hexane/EtOAc (15:1) to give $2(252 \mathrm{mg}, 82 \%)$ as yellow solid $\mathrm{mp} 214-215^{\circ} \mathrm{C}$. ${ }^{1} \mathrm{HNMR}\left(300 \mathrm{MHz}, \mathrm{CDCl}_{3}\right) \delta 8.22(1 \mathrm{H}, \mathrm{m}), 7.61(1 \mathrm{H}, \mathrm{m}), 7.32$ $(1 \mathrm{H}, \mathrm{m}), 7.20(1 \mathrm{H}, \mathrm{m}), 6.47(1 \mathrm{H}, \mathrm{d}, J=9.9 \mathrm{~Hz}), 6.16(1 \mathrm{H}, \mathrm{s})$, $5.44(1 \mathrm{H}, \mathrm{d}, J=9.6 \mathrm{~Hz}), 3.81(3 \mathrm{H}, \mathrm{s}), 1.47(6 \mathrm{H}, \mathrm{s}) .{ }^{13} \mathrm{C} \mathrm{NMR}(75$ $\left.\mathrm{MHz}, \mathrm{CDCl}_{3}\right) \delta 181.1,165.2,161.6,144.8,144.3,134.0,126.1$, $122.9,122.1,121.8,121.6,116.2,106.9,101.0,97.8,76.4,43.7$, 27.0. IR (KBr) 3457, 2971, 1631, 1586, 1554, 1468, 1401, 1327 , $1270,1163,762 \mathrm{~cm}^{-1}$. EI-HRMS $m / z(\mathrm{M})^{+}$calcd for $\mathrm{C}_{19} \mathrm{H}_{17} \mathrm{NO}_{3}$ : 307.1208. Found: 307.1210.

Compound 6. To a solution of $\mathbf{1}(321 \mathrm{mg}, 1 \mathrm{mmol})$ in ethyl acetate $(10 \mathrm{~mL})$ was added catalytic amount of $\mathrm{Pd} / \mathrm{C}(10 \%)$ and hydrogenated (30 Psi) by using autoclave for $1 \mathrm{~h}$. The reaction mixture was filtered through celite and removal of the solvent at reduced pressure left yellow colored solid, which was then purified by column chromatography on silica gel with hexane/ EtOAc (1:1) to give $\mathbf{6}(259 \mathrm{mg}, 80 \%)$ as pale yellow solid. $\mathrm{mp}$ $134-135^{\circ} \mathrm{C} .{ }^{1} \mathrm{H}$ NMR $\left(300 \mathrm{MHz}, \mathrm{CDCl}_{3}\right) \delta 8.22(1 \mathrm{H}, \mathrm{m}), 7.47$ $(1 \mathrm{H}, \mathrm{m}), 7.21(1 \mathrm{H}, \mathrm{m}), 7.09(1 \mathrm{H}, \mathrm{m}), 6.21(1 \mathrm{H}, \mathrm{s}), 3.85(3 \mathrm{H}, \mathrm{s})$, $3.66(3 \mathrm{H}, \mathrm{s}), 2.75(2 \mathrm{H}, \mathrm{t}, J=6.3 \mathrm{~Hz}), 1.66(2 \mathrm{H}, \mathrm{t}, J=6.3 \mathrm{~Hz})$, $1.35(6 \mathrm{H}, \mathrm{s}) .{ }^{13} \mathrm{C} \mathrm{NMR}\left(75 \mathrm{MHz}, \mathrm{CDCl}_{3}\right) \delta 177.9,160.5,159.6$, 150.2, 145.9, 132.4, 126.9, 125.8, 121.6, 116.3, 110.9, 101.5, 95.5, 75.1, 56.1, 44.2, 33.2, 26.8, 22.9. IR (KBr) 2969, 1631, $1590,1548,1476,1398,1332,1271,1146,754 \mathrm{~cm}^{-1}$. HRMS $m / z(\mathrm{M})^{+}$calcd for $\mathrm{C}_{20} \mathrm{H}_{21} \mathrm{NO}_{3}: 323.1521$. Found: 323.1520 .

Compound 7. To a solution of $2(153.5 \mathrm{mg}, 0.5 \mathrm{mmol})$ in ethyl acetate $(7 \mathrm{~mL})$ was added catalytic amount of $\mathrm{Pd} / \mathrm{C}(10 \%)$ and hydrogenated (30 Psi) by using autoclave for $1 \mathrm{~h}$. The reaction mixture was filtered through celite and removal of the solvent at reduced pressure left yellow colored solid, which was then purified by column chromatography on silica gel with hexane/EtOAc (10:1) to give $7(127 \mathrm{mg}, 82 \%)$ as yellow solid. mp $217-218{ }^{\circ} \mathrm{C} .{ }^{1} \mathrm{H}$ NMR $\left(300 \mathrm{MHz}, \mathrm{CDCl}_{3}\right) \delta 8.22(1 \mathrm{H}, \mathrm{d}$, $J=7.8 \mathrm{~Hz}), 7.59(1 \mathrm{H}, \mathrm{t}, J=6.9 \mathrm{~Hz}), 7.32(1 \mathrm{H}, \mathrm{d}, J=8.4 \mathrm{~Hz})$, $7.16(1 \mathrm{H}, \mathrm{t}, J=5.7 \mathrm{~Hz}), 6.11(1 \mathrm{H}, \mathrm{s}), 3.77(\mathrm{~s}, 3 \mathrm{H}), 2.80(2 \mathrm{H}, \mathrm{t}$, $J=6.0 \mathrm{~Hz}), 1.66(2 \mathrm{H}, \mathrm{t}, J=6.3 \mathrm{~Hz}), 1.35(6 \mathrm{H}, \mathrm{s}) .{ }^{13} \mathrm{C} \mathrm{NMR}(75$ $\left.\mathrm{MHz}, \mathrm{CDCl}_{3}\right) \delta 181.4,162.5,162.0,148.0,146.3,133.8,126.1$, $122.2,121.8,116.6,107.3,99.4,98.9,75.2,43.9,33.3,26.8$, 23.1. IR (KBr) 3469, 2961, 1630, 1574, 1475, 1396, 1328, 1265 , $1155,757 \mathrm{~cm}^{-1}$. EI-HRMS $m / z(\mathrm{M})^{+}$calcd for $\mathrm{C}_{19} \mathrm{H}_{19} \mathrm{NO}_{3}$ : 309.1365. Found: 309.1363.

Compound 8. To a solution of $4(567.5 \mathrm{mg}, 2.5 \mathrm{mmol})$ in THF $(10 \mathrm{~mL})$ was added citral $(1140 \mathrm{mg}, 7.5 \mathrm{mmol})$, EDDA (90 $\mathrm{mg}, 0.5 \mathrm{mmol}$ ) and the reaction mixture was refluxed under nitrogen atmosphere for $10 \mathrm{~h}$. Removal of the solvent at reduced pressure left dark colored semi solid, which was then purified by column chromatography on silica gel with hexane/EtOAc $(10: 1)$ to give $8(722 \mathrm{mg}, 80 \%)$ as yellow solid. mp $196-197^{\circ} \mathrm{C}$. ${ }^{1} \mathrm{H}$ NMR $\left(300 \mathrm{MHz}\right.$, Acetone- $\left.d_{6}\right) \delta 10.24(1 \mathrm{H}, \mathrm{s}), 8.23-8.20$ $(1 \mathrm{H}, \mathrm{m}), 7.68-7.61(2 \mathrm{H}, \mathrm{m}), 7.27-7.21(1 \mathrm{H}, \mathrm{m}), 6.96(1 \mathrm{H}, \mathrm{d}, J=$ $9.9 \mathrm{~Hz}), 6.02(1 \mathrm{H}, \mathrm{s}), 5.62(1 \mathrm{H}, \mathrm{d}, J=10.2 \mathrm{~Hz}), 5.07(1 \mathrm{H}, \mathrm{t}, J=$ $5.7 \mathrm{~Hz}), 2.14-2.06(2 \mathrm{H}, \mathrm{m}), 1.75-1.66(2 \mathrm{H}, \mathrm{m}), 1.46(3 \mathrm{H}, \mathrm{s}), 1.39$ $(3 \mathrm{H}, \mathrm{s}), 1.26(3 \mathrm{H}, \mathrm{s}) .{ }^{13} \mathrm{C}$ NMR $\left(75 \mathrm{MHz}\right.$, Acetone- $\left.d_{6}\right) \delta 182.1$ 165.8, 161.0, 141.9, 138.8, 134.7, 132.1, 126.2, 125.8, 124.8, $122.6,120.5,118.1,116.8,105.2,98.7,97.3,80.3,41.7,26.7$, $25.8,23.3,17.7$. IR (KBr) 3439, 2948, 1647, 1610, 1542, 1475, $1333,1156,758 \mathrm{~cm}^{-1}$. HRMS $m / z(\mathrm{M})^{+}$calcd for $\mathrm{C}_{23} \mathrm{H}_{23} \mathrm{NO}_{3}$ : 361.1678. Found: 361.1680.

Compound 9. To a solution of $4(567.5 \mathrm{mg}, 2.5 \mathrm{mmol})$ in THF $(10 \mathrm{~mL})$ was added farnesal (1650 mg, $7.5 \mathrm{mmol})$, EDDA (90 $\mathrm{mg}, 0.5 \mathrm{mmol}$ ) and the reaction mixture was refluxed under nitrogen atmosphere for $12 \mathrm{~h}$. Removal of the solvent at reduced pressure left dark colored semi solid, which was then purified by column chromatography on silica gel with hexane/ EtOAc (10:1) to give 9 (751 mg, 70\%) as yellow solid. mp 170 $171{ }^{\circ} \mathrm{C} .{ }^{1} \mathrm{H}$ NMR $\left(300 \mathrm{MHz}\right.$, Acetone- $\left.d_{6}\right) \delta 10.28(1 \mathrm{H}, \mathrm{s}), 8.26$ $(1 \mathrm{H}, \mathrm{m}), 7.71-7.69(2 \mathrm{H}, \mathrm{m}), 7.29(1 \mathrm{H}, \mathrm{m}), 7.01(1 \mathrm{H}, \mathrm{d}, J=$ $10.2 \mathrm{~Hz}), 6.07(1 \mathrm{H}, \mathrm{s}), 5.88(1 \mathrm{H}, \mathrm{d}, J=10.5 \mathrm{~Hz}), 5.15(1 \mathrm{H}, \mathrm{t}$, $J=7.5 \mathrm{~Hz}), 5.07(1 \mathrm{H}, \mathrm{t}, J=7.8 \mathrm{~Hz}), 2.19-2.11(2 \mathrm{H}, \mathrm{m}), 2.08-$ $2.03(2 \mathrm{H}, \mathrm{m}), 1.97-1.92(2 \mathrm{H}, \mathrm{m}), 1.80-1.74(2 \mathrm{H}, \mathrm{m}), 1.62(3 \mathrm{H}$, $\mathrm{s}), 1.57(3 \mathrm{H}, \mathrm{s}), 1.56(3 \mathrm{H}, \mathrm{s}), 1.44(3 \mathrm{H}, \mathrm{s}) .{ }^{13} \mathrm{C} \mathrm{NMR}(75 \mathrm{MHz}$, Acetone- $\left.d_{6}\right) \delta 182.2,165.8,161.1,142.0,138.9,135.9,134.7$, $131.7,126.3,125.9,125.1,124.8,122.7,120.6,118.1,116.8$, 105.3, 98.8, 97.4, 80.4, 41.8, 40.4, 27.4, 26.7, 25.8, 23.3, 17.7, 16.1. IR (KBr) 3321, 2920, 1646, 1600, 1539, 1479, 1370, 1275, $1160,753 \mathrm{~cm}^{-1}$. EI-HRMS $m / z(\mathrm{M})^{+}$calcd for $\mathrm{C}_{28} \mathrm{H}_{31} \mathrm{NO}_{3}$ : 429.2304. Found: 429.2303.

Compound 10. To a solution of $8(361 \mathrm{mg}, 1 \mathrm{mmol})$ in acetone $(15 \mathrm{~mL})$ was added iodomethane $(1.42 \mathrm{~g}, 10 \mathrm{mmol})$, potassium carbonate $(1.38 \mathrm{~g}, 10 \mathrm{mmol})$ and the reaction mixture was refluxed under nitrogen atmosphere for $2 \mathrm{~h}$. The reaction mixture was filtered and removal of the solvent at reduced pressure left yellow colored solid, which was then purified by column chromatography on silica gel with hexane/EtOAc (1:1) 
to give 10 (335 mg, 86\%) as yellow solid. mp $79-80{ }^{\circ} \mathrm{C} .{ }^{1} \mathrm{H}$ NMR $\left(300 \mathrm{MHz}\right.$, Acetone- $\left.d_{6}\right) \delta 8.18(1 \mathrm{H}, \mathrm{m}), 7.64(1 \mathrm{H}, \mathrm{m}), 7.50$ $(1 \mathrm{H}, \mathrm{m}), 7.21(1 \mathrm{H}, \mathrm{m}), 6.75(1 \mathrm{H}, \mathrm{d}, J=9.6 \mathrm{~Hz}), 6.34(1 \mathrm{H}, \mathrm{s})$, $5.61(1 \mathrm{H}, \mathrm{d}, J=9.6 \mathrm{~Hz}), 5.13(1 \mathrm{H}, \mathrm{t}, J=5.7 \mathrm{~Hz}), 3.89(3 \mathrm{H}, \mathrm{s})$, $3.87(3 \mathrm{H}, \mathrm{s}), 2.20-2.11(2 \mathrm{H}, \mathrm{m}), 1.87-1.82(2 \mathrm{H}, \mathrm{m}), 1.64(3 \mathrm{H}$, s), $1.58(3 \mathrm{H}, \mathrm{s}), 1.50(3 \mathrm{H}, \mathrm{s}) .{ }^{13} \mathrm{C} \mathrm{NMR}\left(75 \mathrm{MHz}\right.$, Acetone- $\left.d_{6}\right) \delta$ $176.4,163.8,160.1,147.4,145.7,133.3,132.2$, 127.1, 126.4, $124.9,123.1,122.3,117.6,111.3,104.1,95.0,79.3,56.4,44.8$, 40.5, 25.8, 25.2, 23.4, 17.7. IR (KBr) 2966, 2919, 1649, 1600, $1540,1477,1429,1381,1282,1159,753 \mathrm{~cm}^{-1}$. HRMS m/z (M) calcd for $\mathrm{C}_{25} \mathrm{H}_{27} \mathrm{NO}_{3}$ : 389.1991 . Found: 389.1993 .

Compound 11. To a solution of $9(429 \mathrm{mg}, 1 \mathrm{mmol})$ in acetone $(15 \mathrm{~mL})$ was added iodomethane $(1.42 \mathrm{~g}, 10 \mathrm{mmol})$, potassium carbonate $(1.38 \mathrm{~g}, 10 \mathrm{mmol})$ and the reaction mixture was refluxed under nitrogen atmosphere for $2 \mathrm{~h}$. The reaction mixture was filtered and removal of the solvent at reduced pressure left yellow colored solid, which was then purified by column chromatography on silica gel with hexane/EtOAc $(1: 1)$ to give $\mathbf{1 1}$ (320 mg, 70\%) as yellow semi solid. ${ }^{1} \mathrm{H}$ NMR (300 $\mathrm{MHz}$, Acetone- $\left.d_{6}\right) \delta 8.11(1 \mathrm{H}, \mathrm{m}), 7.55(1 \mathrm{H}, \mathrm{m}), 7.38(1 \mathrm{H}, \mathrm{m})$, $7.10(1 \mathrm{H}, \mathrm{m}), 6.64(1 \mathrm{H}, \mathrm{d}, J=9.6 \mathrm{~Hz}), 6.25(1 \mathrm{H}, \mathrm{s}), 5.51(1 \mathrm{H}$, $\mathrm{d}, J=9.6 \mathrm{~Hz}), 5.08(1 \mathrm{H}, \mathrm{t}, J=6.9 \mathrm{~Hz}), 4.99(1 \mathrm{H}, \mathrm{t}, J=6.3 \mathrm{~Hz})$, $3.80(3 \mathrm{H}, \mathrm{s}), 3.75(3 \mathrm{H}, \mathrm{s}), 2.12-2.05(2 \mathrm{H}, \mathrm{m}), 1.97-1.93(2 \mathrm{H}, \mathrm{m})$, $1.90-1.85(2 \mathrm{H}, \mathrm{m}), 1.79-1.74(2 \mathrm{H}, \mathrm{m}), 1.54(3 \mathrm{H}, \mathrm{s}), 1.51(3 \mathrm{H}$, s), $1.48(3 \mathrm{H}, \mathrm{s}), 1.40(3 \mathrm{H}, \mathrm{s}) .{ }^{13} \mathrm{C}$ NMR $\left(75 \mathrm{MHz}\right.$, Acetone- $\left.d_{6}\right)$ $\delta 176.4,163.7,160.0,147.6,145.7,135.9,133.2,131.7,127.1$, $126.4,125.1,124.8,123.1,123.0,122.2,117.5,111.2,104.0$, 95.0, 79.2, 56.3, 44.8, 40.5, 40.4, 27.4, 25.9, 25.2, 23.3, 17.8, 16.1. IR (KBr) 2956, 2923, 1618, 1490, 1388, 1208, 1140, 757 $\mathrm{cm}^{-1}$. EI-HRMS m/z $(\mathrm{M})^{+}$calcd for $\mathrm{C}_{30} \mathrm{H}_{35} \mathrm{NO}_{3}$ : 457.2617. Found: 457.2614.

Compound 12. To a solution of $\mathbf{8}(361 \mathrm{mg}, 1 \mathrm{mmol})$ in ethyl acetate $(10 \mathrm{~mL})$ was added catalytic amount of $\mathrm{Pd} / \mathrm{C}(10 \%)$ and hydrogenated (30 Psi) by using autoclave for $4 \mathrm{~h}$. The reaction mixture was filtered through celite and removal of the solvent at reduced pressure left yellow colored solid, which was then purified by column chromatography on silica gel with hexane/EtOAc (2:1) to give $\mathbf{1 2}(325 \mathrm{mg}, 89 \%)$ as yellow solid mp $189-190{ }^{\circ} \mathrm{C} .{ }^{1} \mathrm{H}$ NMR $\left(300 \mathrm{MHz}, \mathrm{CDCl}_{3}\right) \delta 8.23(1 \mathrm{H}, \mathrm{m})$, $7.52(1 \mathrm{H}, \mathrm{m}), 7.22-7.12(2 \mathrm{H}, \mathrm{m}), 6.06(1 \mathrm{H}, \mathrm{s}), 2.59-2.55(2 \mathrm{H}$, m), 1.95-1.80 $(2 \mathrm{H}, \mathrm{m}), 1.60-1.43(4 \mathrm{H}, \mathrm{m}), 1.34(1 \mathrm{H}, \mathrm{m}), 1.24$ $(3 \mathrm{H}, \mathrm{s}), 1.14-.106(2 \mathrm{H}, \mathrm{m}), 0.80(6 \mathrm{H}, \mathrm{d}, J=6.6 \mathrm{~Hz}) .{ }^{13} \mathrm{C} \mathrm{NMR}$ $\left(75 \mathrm{MHz} \mathrm{CDCl}_{3}\right) \delta 181.3,162.3,160.5,140.1,140.0,133.6$, 126.3, 122.2, 120.4, 116.4, 104.7, 97.8, 94.8, 77.5, 39.7, 39.4, 30.2 , 28.0, 24.0, 22.7, 21.5, 16.8. IR (KBr) 3429, 2929, 1629, $1598,1493,1394,1205,1105,1034,759 \mathrm{~cm}^{-1}$. HRMS $m / z(\mathrm{M})^{+}$ calcd for $\mathrm{C}_{23} \mathrm{H}_{27} \mathrm{NO}_{3}$ : 365.1991. Found: 365.1993 .

Compound 13. To a solution of $\mathbf{1 0}(194.5 \mathrm{mg}, 0.5 \mathrm{mmol})$ in ethyl acetate $(10 \mathrm{~mL})$ was added catalytic amount of $\mathrm{Pd} / \mathrm{C}(10 \%)$ and hydrogenated (30 Psi) by using autoclave for $4 \mathrm{~h}$. The reaction mixture was filtered through celite and removal of the solvent at reduced pressure left yellow colored solid, which was then purified by column chromatography on silica gel with hexane/EtOAc (1:1) to give $\mathbf{1 3}(153.3 \mathrm{mg}, 78 \%)$ as pale yellow semi solid. ${ }^{1} \mathrm{H}$ NMR $\left(300 \mathrm{MHz}, \mathrm{CDCl}_{3}\right) \delta 8.22(1 \mathrm{H}, \mathrm{m}), 7.47$ $(1 \mathrm{H}, \mathrm{m}), 7.22(1 \mathrm{H}, \mathrm{m}), 7.08(1 \mathrm{H}, \mathrm{m}), 6.16(1 \mathrm{H}, \mathrm{s}), 3.85(3 \mathrm{H}, \mathrm{s})$, $3.66(3 \mathrm{H}, \mathrm{s}), 2.75-2.70(2 \mathrm{H}, \mathrm{m}), 1.68-1.48(5 \mathrm{H}, \mathrm{m}), 1.31(3 \mathrm{H}, \mathrm{s})$, $1.61-1.10(4 \mathrm{H}, \mathrm{m}), 0.81(6 \mathrm{H}, \mathrm{d}, J=6.6 \mathrm{~Hz}) .{ }^{13} \mathrm{C} \mathrm{NMR}(75 \mathrm{MHz}$, $\left.\mathrm{CDCl}_{3}\right) \delta 177.9,160.5,159.7,150.1,145.9,132.4,126.9,125.8$,
$121.5,116.3,110.8,101.8,95.6,77.2,56.1,44.2,40.1,39.4$, 31.3, 27.9, 23.9, 22.8, 22.7, 22.6, 21.4. IR (KBr) 2944, 1595, $1479,1388,1325,1138,753 \mathrm{~cm}^{-1}$. HRMS $m / z(\mathrm{M})^{+}$calcd for $\mathrm{C}_{25} \mathrm{H}_{31} \mathrm{NO}_{3}$ : 393.2304. Found: 393.2303.

Acknowledgments. This work was supported by a Yeungnam University research grant, 209-A-380-024.

\section{References}

1. (a) Svoboda, G. H. Lloydia 1966, 29, 206. (b) Svoboda, G. H.; Poore, G. A.; Simpson, P. J.; Boder, G. B. J. Pharm. Sci. 1966, 55, 758.

2. (a) Drexler, C.; Hosseini, M. W.; Pratviel, G.; Meunier, B. Chem. Commun. 1998, 1343. (b) Viola, A.; Mannoni, P.; Chanon, M.; Juliard, M.; Mehta, G.; Maiya, B. G.; Muthusamy, S.; Sambiaah, T. J. Photochem. Photobiol. B 1997, 40, 263.

3. (a) Yamamoto, N.; Furukawa, H.; Ito, Y.; Yoshida, S.; Maeno, K.; Nishiyama, Y. Antiviral Res. 1989, 12, 21. (b) Kramer, M. J.; Cleeland, R.; Grunberg, E. Antimicrob. Agents Chemother. 1976, 9, 233.

4. Shen, Y. C.; Wang, L. T.; Khalil, A. T.; Chiang, L. C.; Cheng, P. W. Chem. Pharm. Bull. 2005, 53, 244.

5. Hay, A. E.; Hélesbeux, J. J.; Duval, O.; LabaRed, M.; Grellier, P.; Richomme, P. Life Sciences 2004, 75, 3077.

6. Takemura, Y.; Ju-ichi, M.; Ito, C.; Furukawa, H.; Tokuda, H. Planta Med. 1995, 61, 366.

7. (a) Govindachari, T. R.; Pau, B. R.; Subramaniam, P. S. Tetrahedron 1996, 22, 3245. (b) Hughes, G, K.; Lahey, F. N.; Price, J. R. Nature 1948, 162, 223 .

8. Tillequin, F.; Michel, S.; Skaltsounis, A.-L. In Alkaloids: Chemical and Biological Perspectives; Pelletier, S. W., Ed.; Elsevier: Amsterdam, 1998; Vol. 12, p 1

9. (a) Dorr, R. T.; Liddil, J. D.; Von Hoff, D. D.; Soble, M.; Osborne, C. K. Cancer Res. 1989, 49, 340. (b) Svoboda, G. H. Lloydia 1966, 29, 206. (c) Svoboda, G. H.; Poore, G. A. Simpson, P. J.; Boder, G. B. J. Pharm. Sci. 1996, 55, 758.

10. Minh, N. T.; Michel, S.; Tillequin, F.; Litaudon, M.; Sevenet, T.; Lallemand, M.-C. Zeitschrift fuer Naturforschung, B: Chemical Sciences 2003, 58, 1234.

11. Su, T.-L.; Watanabe, K. A. In Studies in Natural Products Chemistry; Atta-ur-Rahmann, Ed.; Elsevier: Amsterdam, 1993; Vol. $13, \mathrm{p} 347$

12. Costes, N.; Michel, S.; Tillequin, F.; Koch, M.; Pierré, A.; Atassi, G. J. Nat. Prod. 1999, 62, 490 .

13. Brum-Bousquet, M.; Mitaku, S.; Skaltsounits, A.-L.; Tillequin, F.; Koch, M. Planta Med. 1988, 54, 340.

14. Magiatis, P.; Mitaku, S.; Skaltsounis, A.-L.; Tillequin, F.; Koch, M.; Pierré, A.; Atassi, G. J. Nat. Prod. 1998, 61, 198.

15. (a) Anand, R. C.; Selvapalam, N. A. Chem. Commun. 1996, 199. (b) Longhhead, D. G. J. Org. Chem. 1990, 55, 2245. (c) Hlubucek, J.; Ritchie, E.; Taylor, W. C. Aust. J. Chem. 1970, 23, 1881. (d) Macdonald, P. L.; Robertson, A. V. Australian J. Chem. 1966, 19 , 275. (e) Govindachari, T. R.; Pai, B. R.; Subramaniam, P. S. Tetrahedron 1966, 22, 3245.

16. Nikaido, T.; Ohmoto, T.; Kinoshita, T.; Sankawa, U.; Delle Monache, F.; Botta, B.; Tomimori, T.; Miyaichi, Y.; Shirataki, Y.; Yokoe, I.; Komatsu, M. Chem. Pharm. Bull. 1989, 37, 1392.

17. (a) Lee, Y. R.; Choi, J. H.; Yoon, S. H. Tetrahedron Lett. 2005, 46, 7539. (b) Lee, Y. R.; Lee, W. K.; Noh, S. K.; Lyoo, W. S. Synthesis 2006, 853. (c) Lee, Y. R.; Kim D. H. Synthesis 2006, 603. (d) Lee, Y. R.; Kim, J. H. Synlett 2007, 2232. (e) Wang, X.; Lee, Y. R. Tetrahedron Lett. 2007, 48, 6275. (f) Wang, X.; Lee, Y. R. Synthesis 2007, 3044. (g) Lee, Y. R.; Xia, L. Synthesis 2007, 3240. (h) Lee, Y. R.; Li, X.; Kim, J. H. J. Org. Chem. 2008, 73, 4313. (i) Lee, Y. R.; Xia, L. Tetrahedron Lett. 2008, 49, 3283. (j) Xia, L.; Lee, Y. R. Synlett 2008, 1643. (k) Lee, Y. R.; Hung, T. V. Tetrahedron 2008, 64, 7338. (1) Lee, Y. R.; Xue, wang. Tetrahedron 2009, 65, 10125. (m) Lee, Y. R.; Kim, Y. M.; Kim, S. H. Tetrahedron 2009, 65, 101.

18. Bahar, M. H. Sabata, B. K. Ind. J. Chem., Section B 1987, 26 B(8), 782.

19. Funayama, S.; Borris, R. P.; Cordell, G. A. J. Nat. Prod. 1983, 46, 391. 